International Journal of Modern Physics D

(C) World Scientific Publishing Company

DESY 11-154

\title{
Dark matter Search and the Scalar Quark Contents of the Nucleon
}

\author{
Simon Dinter, Vincent Drach, Karl Jansen \\ NIC, DESY, Platanenallee 6, 15738 Zeuthen, Germany
}

\begin{abstract}
We present lattice QCD simulation results from the European Twisted Mass Collaboration (ETMC) for the light, strange and charm quark contents of the nucleon. These quantities are important ingredients to estimate the cross-section for the detection of WIMPs as Dark Matter candidates. By employing a particular lattice QCD formulation, i.e. twisted mass fermions, accurate results of the light and strange scalar contents of the nucleon can be obtained. In addition, we provide a bound for the charm quark content of the nucleon.
\end{abstract}

Keywords: Lattice QCD, Dark Matter, Quark Content of the Nucleon

\section{Introduction}

Dark Matter is still quite mysterious and its nature not clarified. Quite popular candidates are weakly interacting massive particles (WIMPs) which appear in many models [1. There are a number of experiments that attempt to detect such WIMPs. The underlying mechanism for the WIMP detection is that -due to their assumed large mass- they produce a Higgs boson which in turn can interact with nucleons. What the experiments are -in principle- able to detect then is the recoil energy of the nucleon struck in this way by a WIMP which is flying through the detector.

Since the Higgs boson is a scalar, the proposed interaction of the WIMP and the nucleon is going to occur via the coupling of the Higgs boson to the scalar quark content of the nucleon. The spin independent (SI) elastic cross section for such a process reads [2]

$$
\sigma_{\mathrm{SI}, \chi \mathrm{N}} \sim\left|\sum_{q_{f}} G_{q_{f}}\left(m_{\chi}^{2}\right)\left\langle N\left|\bar{q}_{f} q_{f}\right| N\right\rangle\right|^{2}
$$

where $G_{q_{f}}$ is the effective coupling constant between a quark of flavour $f$ and the WIMP of mass $m_{\chi}$ and $\left\langle N\left|\bar{q}_{f} q_{f}\right| N\right\rangle$ denotes the scalar content of the nucleon belonging to a quark flavour $f$. The strength of the coupling $G_{q_{f}}$ will depend on the particular model it is computed in.

It is important to note that changes of the scalar content of the nucleon by about $10 \%$ can lead to large changes in the cross-section [2, 3] since $\left\langle N\left|\bar{q}_{f} q_{f}\right| N\right\rangle$ appears quadratically in eq. (1). One problem with calculating the cross-section is that the scalar quark contents of the nucleon are only poorly determined. If chiral 
perturbation theory $(\chi \mathrm{PT})$ is used for their calculations one finds for the ratio $y_{N}$ of the strange to the light scalar contents

$$
y_{N} \equiv \frac{2\langle N|\bar{s} s| N\rangle}{\langle N|\bar{u} u+\bar{d} d| N\rangle}
$$

a value of $y_{N}=0.44(13)$ [4. This suggests that actually the strange quark content of the nucleon is surprisingly large. On the other hand, from this $\chi \mathrm{PT}$ evaluation of $y_{N}$ only an accuracy of about $30 \%$ is achieved leading to a large uncertainty in the cross-section itself. Thus, is seems highly desirable to compute $y_{N}$ accurately and test whether indeed the scalar quark content of the nucleon is as large as indicated by $\chi \mathrm{PT}$.

In principle, lattice QCD provides a tool to reach a precise quantitative result for $y_{N}$. However, also in this framework the computation of $y_{N}$ turns out to be very difficult. One way to calculate $y_{N}$ is via the Feynman-Hellman theorem [5] which uses the quark mass dependence of the nucleon mass,

$$
\sigma_{\pi N}=m\langle N|\bar{u} u+\bar{d} d| N\rangle=m \frac{\partial m_{N}}{\partial m} \quad \text { and } \quad \sigma_{s s N}=m_{s}\langle N|\bar{s} s| N\rangle=m_{s} \frac{\partial m_{N}}{\partial m_{s}},
$$

where we denote with $m$ the mass-degenerate light up and down quark masses and with $m_{s}$ the strange quark mass. However, to determine $y_{N}$ taking the numerical derivative requires many simulations at different values of the quark masses. In addition, it is unclear how sensitive the nucleon mass will be on the variation of the quark mass.

A second approach is to compute the scalar quark contents $\left\langle N\left|\bar{q}_{f} q_{f}\right| N\right\rangle$ directly. The disadvantage here is that dis-connected (singlet) contributions are to be computed which are generically very noisy in lattice calculations and need a very large statistics.

In this proceedings contribution we want to report about a lattice determination of $y_{N}$ which uses a particular formulation of lattice QCD, namely the so-called twisted mass fermions taken at maximal twist. What is important for the discussion here is first of all that physical quantities computed within this approach scale to the continuum limit, i.e. sending the lattice spacing $a$ to zero, with a rate that is of $O\left(a^{2}\right)$ leading to a rapid approach towards the continuum limit. Secondly, twisted mass fermions allow for special techniques to compute dis-connected diagrams which give a much better signal to noise ratio than most other lattice formulations of QCD. In this report we will make heavily use of this special property of twisted mass fermions. A third advantage of twisted mass fermions (at maximal twist) is that they allow for a straightforward renormalization of $\left\langle N\left|\bar{q}_{f} q_{f}\right| N\right\rangle$ [ $]$ avoiding any mixing between the bare light, strange and charm quark matrix elements. Although also chiral invariant lattice fermions share the same property, these latter kind of lattice fermions are much more computer time demanding such that twisted mass fermions, from this point of view, are clearly advantageous.

We want to mention that there exist already a number of computations of the strange quark content of the nucleon, see refs. [7-14]. For a review we refer to ref. [4]. 
However, because of the aforementioned reasons, these calculations are affected with rather large statistical errors. It is our goal to improve significantly on this situation and provide a more precise value of the scalar content of the nucleon for the light and strange quarks. In addition, we will give a bound on the charm quark content.

\section{The calculation and the results}

As said above, we use a particular formulation of lattice QCD called twisted mass fermions as proposed by Frezzotti and Rossi, see refs. [15 18. The European Twisted Mass Collaboration (ETMC) -in which this project is embedded- is using these kind of lattice fermions for a number of years now and has obtained many physical results already, see e.g. refs. 1924 and references therein.

In the latest set of simulations, ETMC has incorporated the complete first two quark generations in the simulations, meaning that besides the light up and down quarks also the strange and charm quarks are taken into account as dynamical degrees of freedom [24 26. This is presently worldwide a rather unique setup for lattice simulations and particularly important for the problem addressed here since we want to evaluate the strange and the charm quark contents of the nucleon. In order to compute nucleon scalar matrix elements a nucleon 2-point function is needed which is given by

$$
C_{N, 2 \mathrm{pts}}^{ \pm}\left(t-t_{\mathrm{src}}\right)=\sum_{\vec{x}} \operatorname{tr} \Gamma^{ \pm}\left\langle\mathcal{J}_{N}(x) \overline{\mathcal{J}_{N}}\left(x_{\mathrm{src}}\right)\right\rangle,
$$

where the subscript $N$ refers to the proton or to the neutron states for which the interpolating fields are given by :

$$
\mathcal{J}^{p}=\epsilon^{a b c}\left(u^{a, T} \mathcal{C} \gamma_{5} d^{b}\right) u^{c} \quad \text { and } \quad \mathcal{J}^{n}=\epsilon^{a b c}\left(d^{a, T} \mathcal{C} \gamma_{5} u^{b}\right) d^{c} .
$$

The projectors used are $\Gamma^{ \pm}=\frac{1 \pm \gamma_{0}}{2}$, and $\mathcal{C}$ is the charge conjugation matrix. Using discrete symmetries and anti-periodic boundary conditions in the time direction for the quark fields, we have $C_{N, 2 \mathrm{pts}}^{+}(t)=-C_{N, 2 \mathrm{pts}}^{-}(T-t)$.

The other input for the computation of the scalar quark content of the nucleon is the zero momentum 3-point function

$$
C_{N, 3 \mathrm{pts}}^{ \pm, O_{q}}\left(t_{s}, \Delta t_{\mathrm{op}}\right)=\sum_{\vec{x}, \vec{x}_{\mathrm{op}}} \operatorname{tr} \Gamma^{ \pm}\left\langle\mathcal{J}_{N}(x) O_{q}\left(x_{\mathrm{op}}\right) \overline{\mathcal{J}_{N}}\left(x_{\mathrm{src}}\right)\right\rangle,
$$

where $O_{q}$ is an operator having scalar quantum numbers, $\Delta t_{\mathrm{op}}=t_{\mathrm{op}}-t_{\mathrm{src}}$ is the time of insertion of the operator, and $t_{s}=t-t_{\mathrm{src}}$ gives the so-called sourcesink separation. Since we will consider an operator with a non vanishing vacuum expectation value, we also define

$$
C_{N, 3 \mathrm{pts}}^{ \pm, O_{q}, \mathrm{vev}}\left(t_{s}, \Delta t_{\mathrm{op}}\right)=C_{N, 3 \mathrm{pts}}^{ \pm, O_{q}}\left(t_{s}, \Delta t_{\mathrm{op}}\right)-C_{N, 2 \mathrm{pts}}^{ \pm}\left(t, x_{\mathrm{src}}\right) \sum_{\vec{x}_{\mathrm{op}}}\left\langle O_{q}\left(x_{\mathrm{op}}\right)\right\rangle .
$$

The scalar quark operators $O_{q}$ are given by

$$
O_{l}=\bar{u} u+\bar{d} d, O_{s}=\bar{s} s \quad \text { or } \quad O_{c}=\bar{c} c,
$$


depending on the quantity of interest. Using the definitions of the two- and threepoint functions of Eqs. (4) and (5), we will consider the ratio

$$
R_{O_{q}}\left(t_{s}, t_{\mathrm{op}}\right)=\frac{C_{N, 3 \mathrm{pts}}^{ \pm, O_{q}, \mathrm{vev}}\left(t_{s}, t_{\mathrm{op}}\right)}{C_{N, 2 \mathrm{pts}}^{+}\left(t, x_{\mathrm{src}}\right)}
$$

which is related to $y_{N}$ and will serve as our test quantity for the noise reduction technique to compute dis-connected contributions. Our analysis has been performed on a $32^{3} \times 64$ volume with a lattice spacing of $a=0.0777(4) \mathrm{fm}$ and a pion mass of approximately $380 \mathrm{MeV}$. In order to improve the overlap between the ground state and the interpolating operators we use Gaussian smearing of the quark fields appearing in the interpolating fields. We use APE smearing of the gauge links involved in the Gaussian smearing, following the same strategy as in [27,28.

We will use the powerful variance reduction method for twisted mass fermions introduced in 29,30] and used to study the $\eta^{\prime}$ meson in [31. In Fig. 1, we compare the efficiency of this technique with another, more standard noise reduction technique which relies on the hopping parameter expansion of the Dirac operator. This latter technique can be used for any discretization, and has been introduced in 32 . We refer the interested reader to appendix B. of ref. [30] for the implementation of the method for twisted mass fermions. The here used new variance noise reduction technique improves the signal to noise ratio by a factor $\sim 3$ after having fixed all other parameters. In particular, as can be seen in fig. 1 with a statistics of $O(1000)$ gauge field configurations we can reach a result that is at the $5 \sigma$ level different from zero, while the standard method with such a statistics gives only a result at the $1 \sigma$ level.

Using our improved noise reduction technique for dis-connected contributions, we have first performed an investigation of the light quark content of the nucleon, i.e. $\sigma_{\pi N}$ of eq. (3). Here, it turns out that the connected part of the relevant 3point function are by far dominating and that the dis-connected contribution can be safely neglected. Although such a fact might have been expected, our dedicated investigation is certainly important to confirm this.

To estimate systematic effects coming from the excited states, the final number for $\sigma_{\pi N}$ is then obtained from a distribution of many fit results. From the (weighted) mean and variance we then obtain

$$
\sigma_{\pi N}\left(m_{\mathrm{PS}} \approx 380 \mathrm{MeV}\right)=150(1)(10) \mathrm{MeV},
$$

where the first error is statistical and the second gives the systematic uncertainties. We then proceeded to compute the ratio $R$ of eq. (8) for the strange and charm quark scalar operator. In Fig. 2 (left), we show the plateau corresponding to the strange quark. The plateau value appears to be $5.7 \sigma$ away from zero showing that we indeed can obtain a rather accurate value for the strange quark content of the nucleon.

We also carried out a first study of the charm quark content of the nucleon. Here it is most important that we can use gauge field configurations from $N_{f}=2+1+1$ 


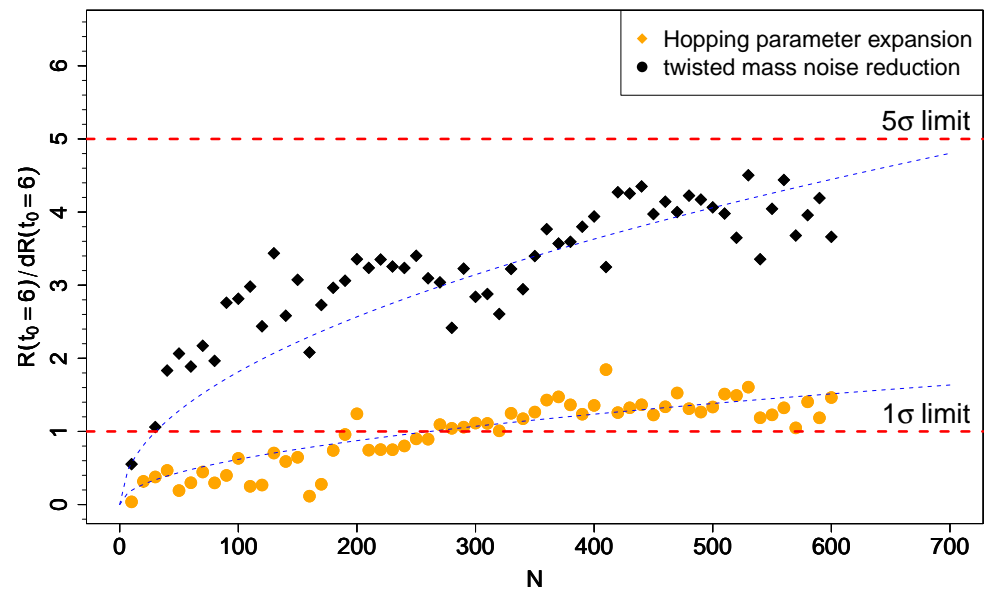

Fig. 1. Signal to noise ratio of the correlator ratio Eq. (8), as a function of the number of configurations used, $N$, for the variance noise reduction technique used in this paper compared to the more standard hopping parameter expansion technique. Note that here the source-sink separation is fixed to $t_{s}=12 a$. Curves are only shown to guide the eyes. Our method allows to reach results at a $\sim 5 \sigma$ significance level with a statistics that is $O(10)$ less than standard methods.
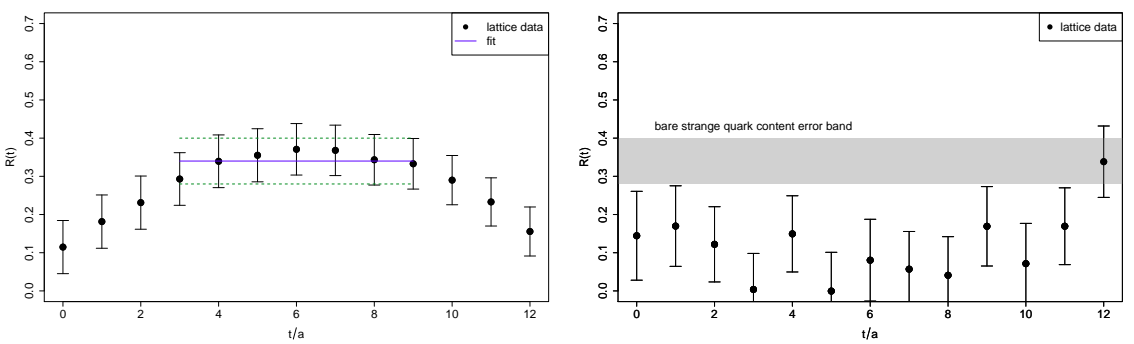

Fig. 2. In the left graph, we plot the bare ratio of Eq. (8) for the strange quark. We used 842 configurations for this analysis. Note that the signal is $5 \sigma$ away from 0 . In the right graph, we show results for the charm content using the same statistics. While certainly no plateau can be identified, the charm quark content is clearly not larger than the strange quark content of the nucleon. As in fig. 1 t $t_{s}=12 a$.

simulations that take into account also the charm quark degree of freedom. We show in Fig. 2 (right) our results for the charm quark. Unfortunately, there is no plateau signal visible. The gray band in Fig. 2 (right) represents our results for the strange quark. The graph then suggests that the charm quark content of the nucleon cannot be large compared to the strange quark as one might expect. 


\section{Conclusion}

In this proceedings contribution we have computed the scalar quark content of the nucleon for all four quarks of the first two generations. We find a value of the phenomenologically important quantity $y_{N}=0.066(11)(2)$, see eq. (2). This value is significantly smaller than predicted in chiral perturbation theory and indicates that the strange quark content is only a few percent. In fig. 3 we give a summary of presently available values for $y_{N}$ demonstrating the precision of our result. We also could provide for the first time a bound for the charm quark content of the nucleon excluding that it is significantly larger than the strange quark content.

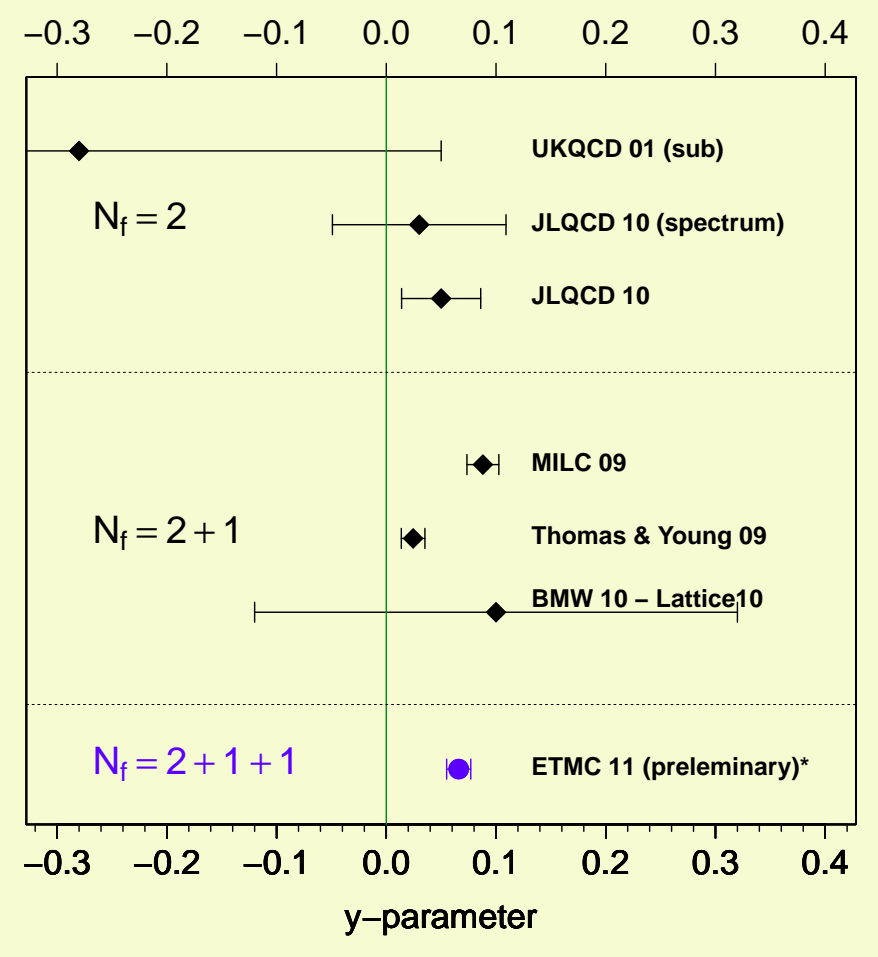

Fig. 3. Compilation of presently available results for the y-parameter $y_{N}$ of eq. (2).

As a present shortcoming, we mention that our calculations are performed at only one value of the lattice spacing, quark mass and volume. Thus we are lacking presently a good estimate of systematic effects originating from varying these parameters of the simulations. Clearly, we will address these systematic errors in future computations which have already been started. 


\section{Acknowledgments}

We thank all members of the ETM Collaboration for a very fruitful collaboration and many valuable discussions. In particular we want to thank R. Frezzotti, G. Herdoiza and G. Rossi for essential discussions about the renormalization aspects. The $\mathrm{HPC}$ resources for this project have been made available by the computer centres of Barcelona, Groningen, Jülich, Lyon, Munich, Paris and Rome (apeNEXT), which we thank for enabling us to perform this work. This work has also been supported in part by the DFG Sonderforschungsbereich/Transregio SFB/TR9-03, and by GENCI (IDRIS - CINES), Grant 2010-052271.

\section{References}

1. Gianfranco Bertone, Dan Hooper, and Joseph Silk. Phys.Rept., 405:279-390, 2005.

2. John R. Ellis, Keith A. Olive, and Christopher Savage. Phys.Rev., D77:065026, 2008.

3. Joel Giedt, Anthony W. Thomas, and Ross D. Young. Phys.Rev.Lett., 103:201802, 2009.

4. Ross D. Young and Anthony W. Thomas. Nucl.Phys., A844:266C-271C, 2010.

5. R. P. Feynman. Phys. Rev., 56:340-343, 1939.

6. S. Dinter et al. in preparation.

7. H. Ohki, S. Aoki, H. Fukaya, S. Hashimoto, T. Kaneko, et al. PoS, LAT2009:124, 2009 .

8. H. Ohki, S. Aoki, H. Fukaya, S. Hashimoto, T. Kaneko, et al. 2010.

9. Sara Collins, Gunnar Bali, Andrea Nobile, Andreas Schafer, Yoshifumi Nakamura, et al. PoS, LATTICE2010:134, 2010.

10. Gunnar Bali, Sara Collins, and Andreas Schafer. PoS, LAT2009:149, 2009

11. Walter Freeman and Doug Toussaint. PoS, LAT2009:137, 2009.

12. S. Durr, Z. Fodor, J. Frison, T. Hemmert, C. Hoelbling, et al. PoS, LATTICE2010:102, 2010.

13. Christopher Michael, C. McNeile, and D. Hepburn. Nucl.Phys.Proc.Suppl., 106:293295, 2002.

14. R.D. Young and A.W. Thomas. Phys.Rev., D81:014503, 2010.

15. Roberto Frezzotti, Pietro Antonio Grassi, Stefan Sint, and Peter Weisz. JHEP, 0108:058, 2001.

16. R. Frezzotti and G. C. Rossi. JHEP, 08:007, 2004.

17. R. Frezzotti and G. C. Rossi. Nucl. Phys. Proc. Suppl., 128:193-202, 2004.

18. R. Frezzotti and G. C. Rossi. JHEP, 10:070, 2004.

19. C. Alexandrou, J. Carbonell, M. Constantinou, P.A. Harraud, P. Guichon, et al. Phys.Rev., D83:114513, 2011.

20. Xu Feng, Karl Jansen, and Dru B. Renner. Phys.Lett., B684:268-274, 2010.

21. Xu Feng, Karl Jansen, and Dru B. Renner. Phys.Rev., D83:094505, 2011.

22. Xu Feng, Karl Jansen, Marcus Petschlies, and Dru B. Renner. 2011.

23. R. Baron et al. JHEP, 1008:097, 2010.

24. R. Baron et al. JHEP, 06:111, 2010.

25. T. Chiarappa et al. Eur. Phys. J., C50:373-383, 2007.

26. Remi Baron et al. Comput.Phys.Commun., 182:299-316, 2011.

27. Simon Dinter, Constantia Alexandrou, Martha Constantinou, Vincent Drach, Karl Jansen, et al. PoS, LATTICE2010:135, 2010.

28. Vincent Drach, Karl Jansen, Jaume Carbonell, Mauro Papinutto, and Constantia Alexandrou. PoS, LATTICE2010:101, 2010. 
November 24, $2011 \quad$ 1:13 WSPC/INSTRUCTION FILE $\quad$ paper

\section{Karl Jansen}

29. Christopher Michael and Carsten Urbach. PoS, LAT2007:122, 2007.

30. Ph. Boucaud et al. Comput. Phys. Commun., 179:695-715, 2008.

31. K. Jansen, Christopher Michael, and C. Urbach. Eur.Phys.J., C58:261-269, 2008.

32. Craig McNeile and Christopher Michael. Phys.Rev., D63:114503, 2001. 